\title{
BIO.I9 - Development of a new molecular tool for antibody detection and purification
}

Franciane Batista ${ }^{1}$; Leonardo Antonio Fernandes ${ }^{1}$; Lina Maria Salazar Echeverri ${ }^{1}$; Gustavo Felippe da Silva ${ }^{1}$; Maria de Lourdes Borba Magalhães ${ }^{1 *}$.

1Outros.

Introduction: Primary antibody detection and purification are crucial procedures in research, diagnosis and therapeutics. Molecular tools currently available consist of secondary antibodies as well as bacterial proteins such as Proteins A and G. Disadvantages of the current methods include complex and expensive production of secondary antibodies. In the case of Proteins A and $\mathrm{G}$, they either do not recognize all IgG isotypes or require acidic elution conditions for antibody purification, which may lead to antibody denaturation. Recently, an IgG binding protein was discovered and its use to produce a chimeric protein fused to streptavidin, named YT1902, is of great impact to detect and purify IgGs, being an attractive strategy to overcome current limitations.

Objective: To develop a new molecular tool for primary antibody detection using chimeric bacterial protein (YT1902) comprising of an IgG binding module linked to enzymatic probes. To develop a chromatographic column for antibody purification using novel chimeric bacterial protein (YT1902).

Methodology: Coding gene of YT1902 protein (Trim21-Streptavidin) was purchased from Genescript and cloned into $\mathrm{pET} 15 \mathrm{~b}(+)$ vector. The resultant recombinant vectors were expressed into E. coli BL21 (DE3) PlysS and induced at $17 \mathrm{oC}$ during 24 hours in the presence of $0.01 \mathrm{mM}$ IPTG. YT1902 was present in inclusion bodies and refolding was performed by washing steps followed by aggregates dissolution with 6M Guanidine Hydrochloride. Refolding was performed by dialysis followed by affinity purification using Ni-NTA resin.

Immunoassays. ELISA was performed by coating on 96 wells microtiters with serum from different animal species or purified IgGs. After blocking with 1\% skim milk, HRP labeled YT1902 was used to detect immobilized IgGs. Western blotting analysis was performed using HRP of Alkaline fosfatase labeled YT1902.

Results: We have produced chimeric proteins, using an IgG binding protein recently discovered (TRIM21) linked to streptavidin. TRIM21 is a cytosolic protein that binds all IgG isotypes from many species. Bacterial expression, purification and refolding were successful. YT1902 was incubated with biotinylated HRP or alkaline phosphatase for immunoassays. ELISA, western blotting and immunohistochemistry experiments demonstrated that the chimeric protein efficiently detected monoclonal and polyclonal antibodies from a wide range of species, including human, mouse, rat, dog, horse and bovine.Purification experiments are underway using biotin agarose and immobilized YT1902 for antibody purification.

Conclusion: YT1902 is able to detect efficiently IgGs from a wide range of mammalian species. The production costs of such proteins is considerable lower than current secondary antibody production. IgG purification using this strategy, will likely be superior than Protein A or G, since elution is not dependent on $\mathrm{pH}$ change, which often cause antibody denaturation. The patent of the current invention has been deposited and will provide a great tool for antibody detection and purification from Horse, Humans, Dogs, Pigs and Mice.

Keywords: antibody detection; antibody purification; immunoassays 